\title{
Metabolic profiling detects biomarkers of protein degradation in COPD patients
}

\author{
Baljit K. Ubhi*, John H. Riley\#, Paul A. Shaw ${ }^{\uparrow}$, David A. Lomas ${ }^{+}$, Ruth Tal-Singer ${ }^{\S}$, \\ William MacNee ${ }^{f}$, Julian L. Griffin* and Susan C. Connor*
}

ABSTRACT: There is a paucity of biomarkers for chronic obstructive pulmonary disease (COPD). Metabolomics were applied to a defined COPD patient cohort from the ECLIPSE study (Evaluation of COPD Longitudinally to Identify Predictive Surrogate End-points). Results were correlated with accepted biomarkers for the disease.

Baseline control serum $(n=66)$ and Global Initiative for Chronic Obstructive Lung Disease (GOLD) stage II $(n=70)$, III $(n=64)$ and IV $(n=44)$ COPD patients were analysed by proton nuclear magnetic resonance $\left({ }^{1} \mathrm{H}\right.$ NMR). Liquid chromatography with tandem mass spectrometry (LC-MS/ MS) was used to confirm amino acid changes detected by ${ }^{1} \mathrm{H}$ NMR. Data were correlated with body composition, emphysema and systemic inflammation.

${ }^{1} \mathrm{H}$ NMR identified decreased lipoproteins, $\mathrm{N}, \mathrm{N}$-dimethylglycine, and increased glutamine, phenylalanine, 3-methylhistidine and ketone bodies in COPD patients with decreased branchedchain amino acids (BCAAs) observed in GOLD stage IV patients. BCAAs, their degradation products, 3-methylhistidine, ketone bodies, and triglycerides were correlated negatively with cachexia and positively with systemic inflammation. Emphysema patients also displayed decreased serum creatine, glycine and $\mathrm{N}, \mathrm{N}$-dimethylglycine. LC-MS/MS confirmed ${ }^{1} \mathrm{H}$ NMR findings relating to BCAAs, glutamine and 3-methylhistidine in GOLD stage IV patients.

NMR-based metabolomics characterised COPD patients based on systemic effects and lung function parameters. Increased protein turnover occurred in all COPD patients with increased protein degradation in individuals with emphysema and cachexia.

KEYWORDS: Chronic obstructive pulmonary disease, liquid chromatography-mass spectrometry, metabolomics, metabonomics, nuclear magnetic resonance spectroscopy, serum

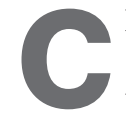

hronic obstructive pulmonary disease (COPD) is a major health burden worldwide, affecting $10-15 \%$ of the adult population aged $\geqslant 40$ yrs [1]. 3 million deaths are caused by COPD annually and it is a major cause of morbidity in an estimated 210 million people worldwide [2]. COPD is the result of chronic airway inflammation that manifests from progressive airflow limitation. Characterisation of COPD patients has relied upon the measurement of airflow limitation, as assessed by forced expiratory volume in $1 \mathrm{~s}$ (FEV1) [3], which has been used to define the condition, its severity and subsequent treatment. However, it is now widely recognised that COPD is a heterogeneous disease with features that are not captured by the measurement of FEV1 [4]. These include systemic inflammation, muscle dysfunction and cardiovascular disease. There is an urgent need for biomarkers that reflect underlying pathological processes giving rise to different components of the COPD phenotype; these biomarkers may predict patient response to different therapies and outcomes in COPD.

Metabolomics is a functional genomic technology that provides a detailed investigation of changes in the metabolic profiles of biofluids, gases (breath) and semi-solids (e.g. intact tissues) [5]. We have used nuclear magnetic resonance (NMR) spectroscopybased metabolomics to detect metabolites in serum that are associated with COPD disease manifestations, thereby serving as potential biomarkers. This was undertaken on samples from a wellcharacterised group of individuals with COPD from the ECLIPSE cohort (Evaluation of COPD Longitudinally to Identify Predictive Surrogate Endpoints) [6]. A targeted analysis using a bespoke liquid chromatography with tandem mass spectrometry

This article has supplementary material accessible from www.erj.ersjournals.com

AFFILIATIONS

*Dept of Biochemistry and

Cambridge Systems Biology Centre University of Cambridge,

${ }^{+}$Dept of Medicine, University of

Cambridge, Cambridge Institute for Medical Research, Cambridge,

\#Respiratory MDC, GlaxoSmithKline, Stevenage,

"Dept of Investigative Preclinical Toxicology, Safety Assessment Division, GlaxoSmithKline R\&D, Ware, and

${ }^{f}$ Centre for Inflammation Research, Queen's Medical Research Institute, Edinburgh, UK.

${ }^{\S}$ GlaxoSmithKline R\&D, King of Prussia, PA, USA.

CORRESPONDENCE

S.C. Connor

Dept of Biochemistry and Cambridge Systems Biology Centre University of Cambridge The Sanger Building 80 Tennis Court Road Cambridge UK E-mail: susan.c.connor@ googlemail.com

Received:

June 302011

Accepted after revision: Nov 152011 First published online: Dec 192011 
(LC-MS/MS) assay of amino acids was also carried out on a subset of the open-profiling metabolomics cohort to analytically validate the NMR results.

\section{MATERIALS AND METHODS \\ Study design}

ECLIPSE is a 3-yr longitudinal observational study in 40-75-yrold current and ex-smoker COPD patients (current/ex-smokers $\mathrm{n}=2,756$ ) and control subjects (current/ex-smokers $\mathrm{n}=343$, nonsmokers $n=223$ ). A full description of the methods, including the computed tomography (CT) scanning procedures and the cohort, have been published $[4,5]$ and are summarised in the online supplementary material.

Serum samples for open-profiling metabolomics $(n=244)$ were obtained from pair-matched control versus COPD subjects based on age, sex and smoking history. All patients and former smoker controls in the metabolomics cohort were ex-smokers to remove the potentially-confounding effects of current smoking. A subgroup of controls who had never smoked was included for comparison with the former smoker controls. Patients were categorised based using the Global Initiative for Chronic Obstructive Lung Disease (GOLD) criteria (described in the online supplementary material): GOLD stages II, III and IV subjects in the NMR cohort showed similar FEV1 to the whole ECLIPSE cohort (table 1). Targeted amino acid analysis used a subset of patients from the original NMR dataset (male smoker control $n=30$, GOLD IV $n=30$ ). Baseline fasting serum samples were obtained from morning blood samples at the initial clinic visit. Diet and over-the-counter pain or prescribed medications were recorded for the previous $24 \mathrm{~h}$.

\section{Open-profiling metabolomics}

High-resolution proton Carr-Purcell-Meiboom-Gill NMR spectra were acquired on a Bruker Avance III NMR spectrometer (699.72 MHz; Bruker BioSpin, Rheinstetten, Germany). Methodological details for sample preparation and spectral acquisition are provided in the online supplementary material. Spectra were pre-processed using Bruker software (Topspin version 1.3 and Amix version 3.7.10; Bruker BioSpin, Karlsruhe, Germany) using a 0.005-ppm bucket width, and referenced and scaled to 4,4-dimethyl-4-silapentane-1-ammoniumtrifluoroacetate [7]. Spectra were assigned using two-dimensional NMR experiments (correlation spectroscopy, total correlation spectroscopy, heteronuclear single-quantum correlation and heteronuclear multiple-bond correlation [8]) and with reference to NMR assignment databases: Chenomx NMR Suite version 4.0 (Chemomx Inc., Edmonton, Canada); Human Metabolome Database (Genome Alberta (Alberta, Canada) and Genome Canada (Ottowa, Canada)); and Biological Magnetic Resonance Bank (BMRB; University of Wisconsin System, Madison, WI, USA) (table S1).

\section{Amino acid analysis}

A quantitative LC-MS/MS amino acid assay was developed to validate the open-profiling metabolomics results. Chloroformate amino acid derivatives (EZFaast; Phenomenex, Macclesfield, UK) were analysed by positive ion electrospray mass spectrometry (MS) using a Waters Acquity UPLC system (Waters Corp., Millford, MA, USA) and Waters Quattro Premier XE triple quadrupole mass spectrometer (Waters Corp.) (table S2). Additional

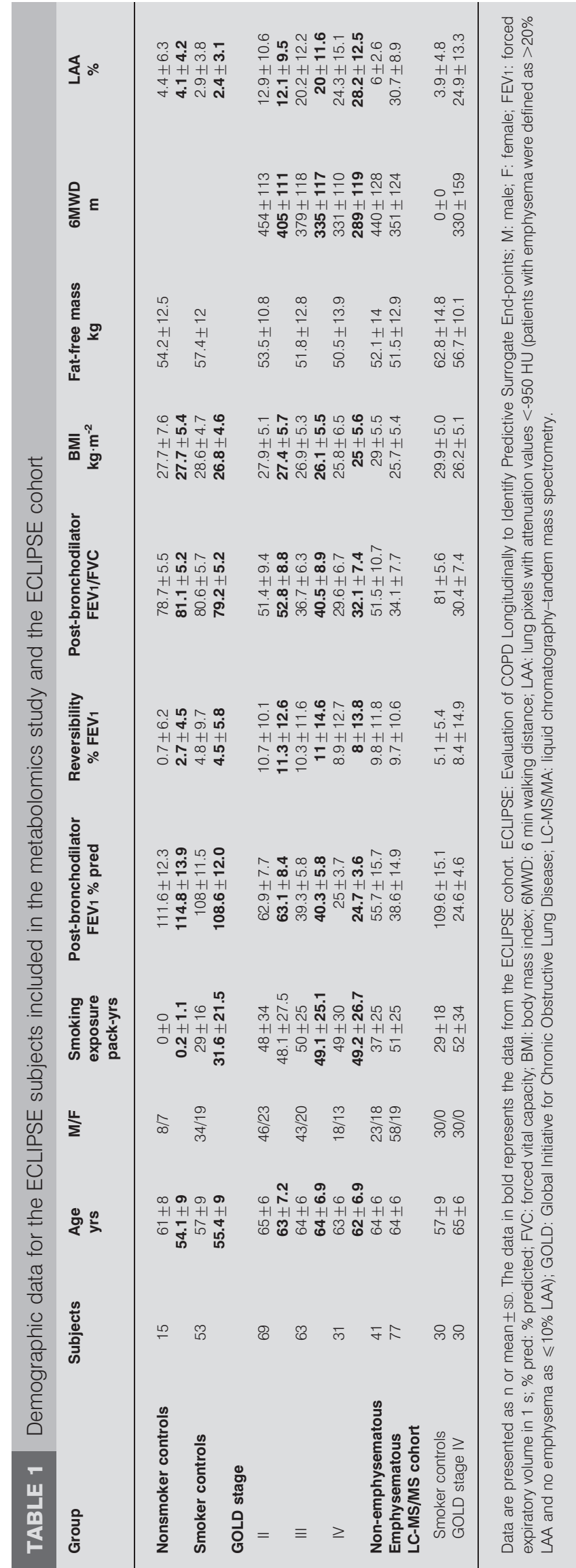


description of the assay methodology and characteristics are described in the online supplementary material.

\section{Statistical analysis}

Outliers and COPD-related effects were investigated using univariate data analysis and multivariate data analysis (MVDA). MVDA methods included unsupervised principal components analysis (PCA), using colour coding to highlight disease and confounding factors (GOLD stage, cachexia, emphysema, diabetes, patient location, age, sex and comorbidities). Using two-thirds of the dataset, supervised partial least squares (PLS) discriminant analysis (DA) were performed to maximise patient group differences (i.e. GOLD versus control, emphysema versus non-emphysema, cachexic versus noncachexic), by rotating PCA components to improve separation among patient groups and highlight key variables. The remaining one-third of the data, "unseen" by the PLS-DA models, was used as independent data to assess the model relevance and error together with any data over-fit.

Statistical significance for individual metabolites was assigned using PLS-DA coefficients or unpaired t-test $\alpha<0.05,<0.1$ and $<0.15$, where $\alpha$ was the false discovery rate (FDR) multiplicity adjustment [9] at the 5, 10 and 15\% level. The NMR data were correlated with physiological and biochemical measurements using PLS and Pearson's correlation. Correlation significance was assigned using the $\mathrm{t}$-distribution for FDR $\alpha<0.05$ and $\leqslant 0.15$, with raw p-values $<0.01$.

\section{RESULTS}

\section{Quality control and outlier detection}

ECLIPSE baseline serum NMR spectra $(n=244)$ were checked for acquisition and processing errors (fig. 1). PCA of processed NMR data was used to observe the overall variation in the data, technical and biological outliers, and sample groupings. Three poorly controlled diabetics were outliers and excluded from analysis. Current smokers $(n=5)$, detected by cotinine assay, were excluded from analysis as low sample numbers precluded adequate analysis of the effects of smoking. Following exclusions, the main variation in principal component (PC) one was from very low-density lipoproteins (VLDLs)/chylomicrons and glucose, probably arising from variable compliance with overnight fasting. The PCA score and loadings plots were dominated by shifting of amino and organic acid resonances in GOLD stage IV COPD patients (fig. S1), consistent with variable serum $\mathrm{pH}$. Further analysis was, therefore, performed on bucketed data summed across individual metabolite regions.

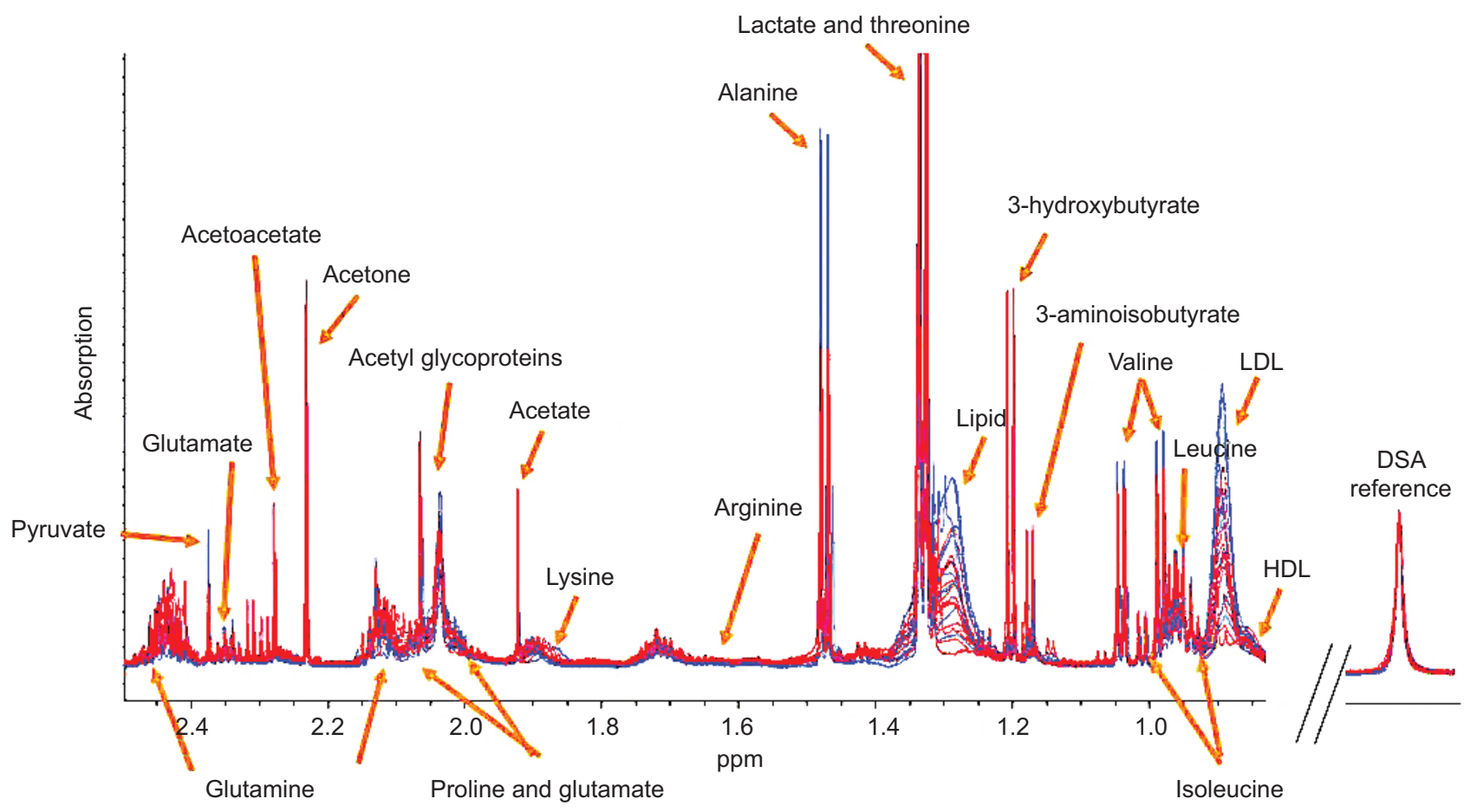

FIGURE 1. Partial 700-MHz ${ }^{1} \mathrm{H}$ nuclear magnetic resonance (NMR) spectra for Global Initiative for Chronic Obstructive Lung Disease stage IV patients (red) versus control subjects (blue). The individual compounds in the spectra were assigned using additional, more detailed NMR experiments (i.e. two-dimensional (2D) NMR: correlation spectroscopy (COSY), total correlation spectroscopy (TOCSY), heteronuclear single-quantum correlation (HSQC) and heteronuclear multiple-bond correlation (HMBC)) applied to ECLIPSE (Evaluation of COPD Longitudinally to Identify Predictive Surrogate End-points) cohort serum samples. These experiments showed which resonances corresponded to protons in the same molecule and provided additional information regarding neighbouring ${ }^{13} \mathrm{C}$ in the same molecule. Using the chemical shift (resonance position) information for ${ }^{1} \mathrm{H}$ and ${ }^{13} \mathrm{C}$ in the same molecule it was possible to assign the NMR signals using existing metabolomic databases. $2 \mathrm{D}$ NMR ${ }^{1} \mathrm{H}-{ }^{1} \mathrm{H}$ COSY and TOCSY experiments highlight interactions between adjacent protons (proton spin-spin coupling). $2 \mathrm{D}{ }^{1} \mathrm{H}-{ }^{13} \mathrm{C} H S Q \mathrm{C}$ and $\mathrm{HMBC}$ experiments showed interactions between protons and their attached ${ }^{13} \mathrm{C}$ or ${ }^{13} \mathrm{C}$ up to three/four bonds away, respectively. Where possible, additional confirmatory evidence was obtained using Chenomx deconvolution software (Chemonix Inc., Edmonton, Canada). LDL: Iow-density lipoproteins; HDL: high-density lipoproteins; DSA: 4,4-dimethyl-4-silapentane-1-ammonium trifluoroacetate. 


\section{Serum metabolite differences between COPD patients and control subjects}

NMR spectra of COPD patients were significantly different from controls by PCA, PLS-DA and t-test (fig. 2 and table 2). The PCA PC2 scores for patients with severe (GOLD stage III) and very severe (GOLD stage IV) COPD, and the majority of patients with moderate (GOLD stage II) COPD clustered separately from the controls (fig. 2). Because PCA is unsupervised (i.e. no prior group knowledge is used in the calculation) the partial clustering observed for COPD patients demonstrates that the second major source of variation in the data (after 33\% of variation due to food intake (PC1)) was due to COPD. The partial COPD-related clustering was caused by increased glutamine, 3-methylhistidine, phenylalanine and dimethylglycine, acetoacetate, and 3-hydroxybutyrate, and decreased unsaturated lipid VLDL. The significant spectral regions containing these metabolites are detailed in the online supplementary material (tables S3-S5).

Supervised MVDA (PLS-DA) was used to maximise this observed difference between groups and the results compared with $t$-test results. The PLS-DA model $\left(R^{2} X=44 \%, R^{2} Y=22 \%\right.$ and $Q^{2}=17 \%$ ) showed similar metabolite changes to those observed by PCA plus increased acetate, monoglyceryl fatty acids, creatine, ascorbate and aspartate, and decreased $O$ acetylated glycoproteins and glycerol (table 2).

\section{Relationship between serum metabolites and disease severity}

No GOLD stage II-related group pattern was observed. PCA of GOLD stages III and IV and control samples showed clustering of patients versus controls subjects (fig. 2). Supervised PLS-DA classification of GOLD stages III and IV versus control subjects, both separately and jointly, produced significant models. In addition to those metabolites highlighted in the patient versus control subjects comparison (table 2), decreased 3-hydroxyisobutyrate, isobutyrate, branched-chain amino acids (BCAAs), methionine, trimethylamine and highdensity lipoprotein (HDL) were also observed in GOLD stage IV patients.

The PLS-DA model for moderate-to-severely affected (GOLD stages III and IV) patients versus controls subjects was generated from two-thirds of the data so that the remaining one-third

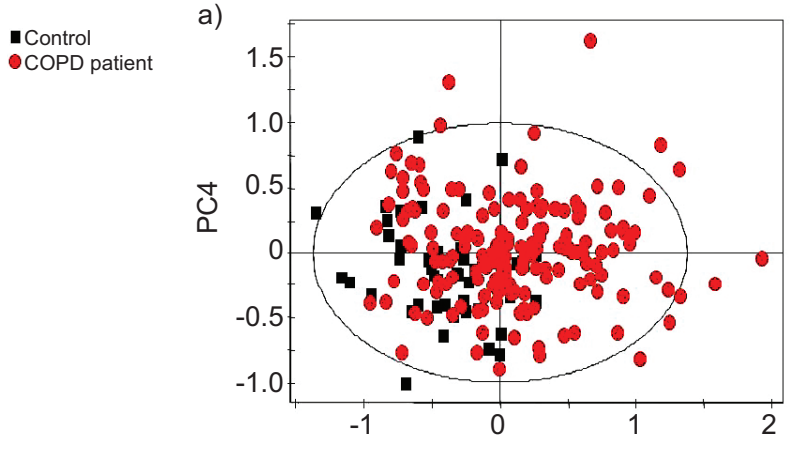

b)
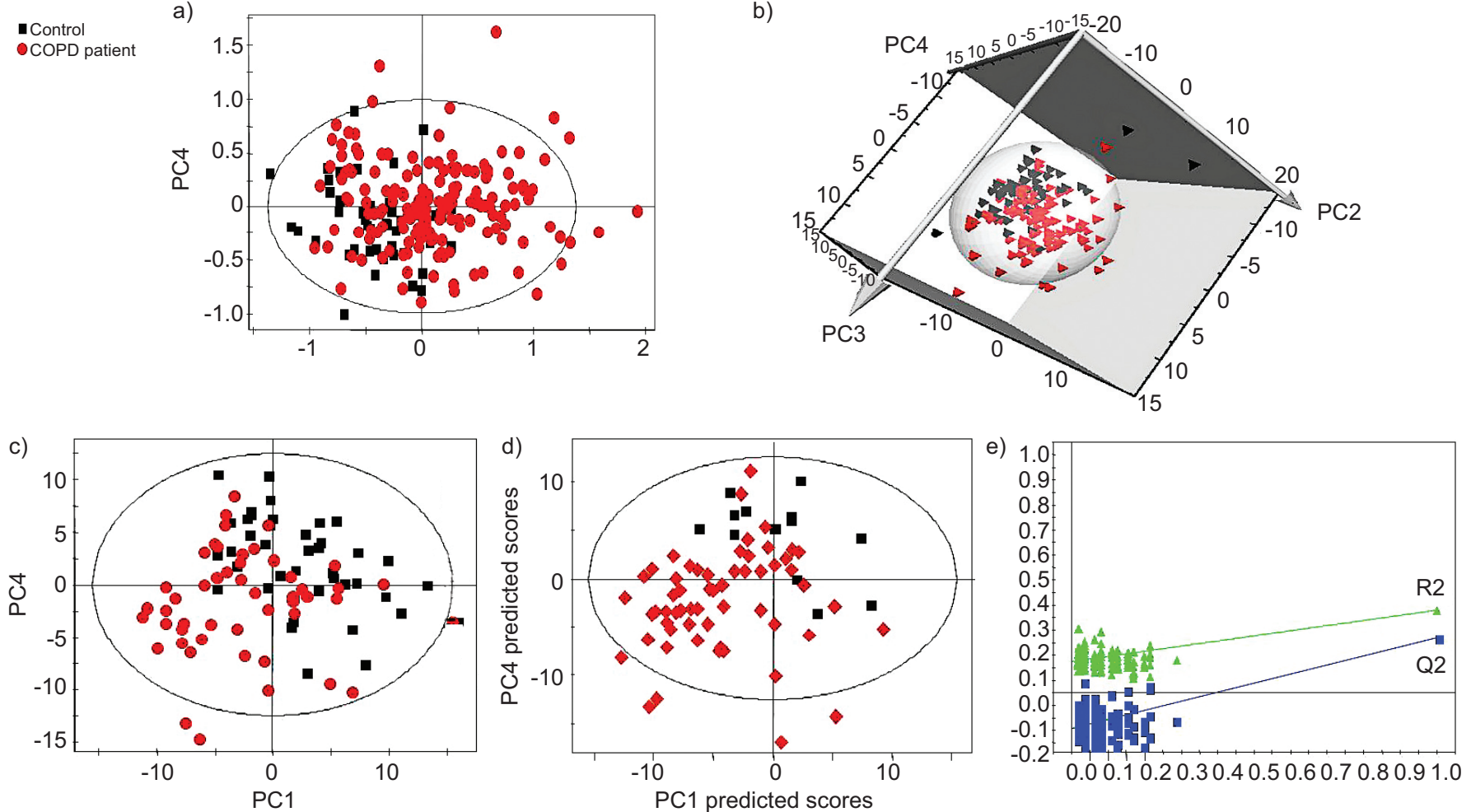

FIGURE 2. Score plots summarising the multivariate analysis of chronic obstructive pulmonary disease (COPD) patients versus control subjects. a) Two-dimensional score plot of principal components (PCs) 2-4 for a principal component analysis (PCA) model of all patients: Global Initiative for Chronic Obstructive Lung Disease (GOLD) stages II, III and IV versus control subjects. The variance explained by the model (i.e. the sum of squares of all the X's explained by the extracted components (R2)) for PC1 was $33.5 \%$ and for PCs $2-4$ was 34.4\%. b) Three-dimensional (3D) PCA score plot of GOLD stages III and IV patients versus control subjects; R2 for PC1 was $38.8 \%$ and for PCs $2-4$ was $34.5 \%$. The variation described by the first component for both a) and b) was mainly due to very low-density lipoproteins and glucose. c-e) Partial least squares (PLS) discriminant analysis (DA) predictive modelling of GOLD stage III plus GOLD stage IV COPD patients versus ex-smoker control subjects (male and female) showing score plots of $\mathrm{c}$ ) the training set, d) the test set and e) random permutation test-validation plot. The training dataset used to generate the model consisted of equal numbers of COPD patients and controls. To test the accuracy of the model and degree of over-fit, the classes of independent samples "unseen" by the original model were predicted to calculate prediction error. The test set samples had fewer controls than patients due to the smaller number of controls in the dataset. A PLS-DA model was generated for 90 control subjects and GOLD stages III and IV patients (training set), and the scores predicted for 71 new samples using the model generated for the training set. New samples were predicted with good accuracy (82\%; model diagnostics were $\mathrm{R} 2 \mathrm{X}=52.2 \%, \mathrm{R} 2 \mathrm{Y}=33 \%$ and $\mathrm{Q} 2=22.2 \%$ ). 


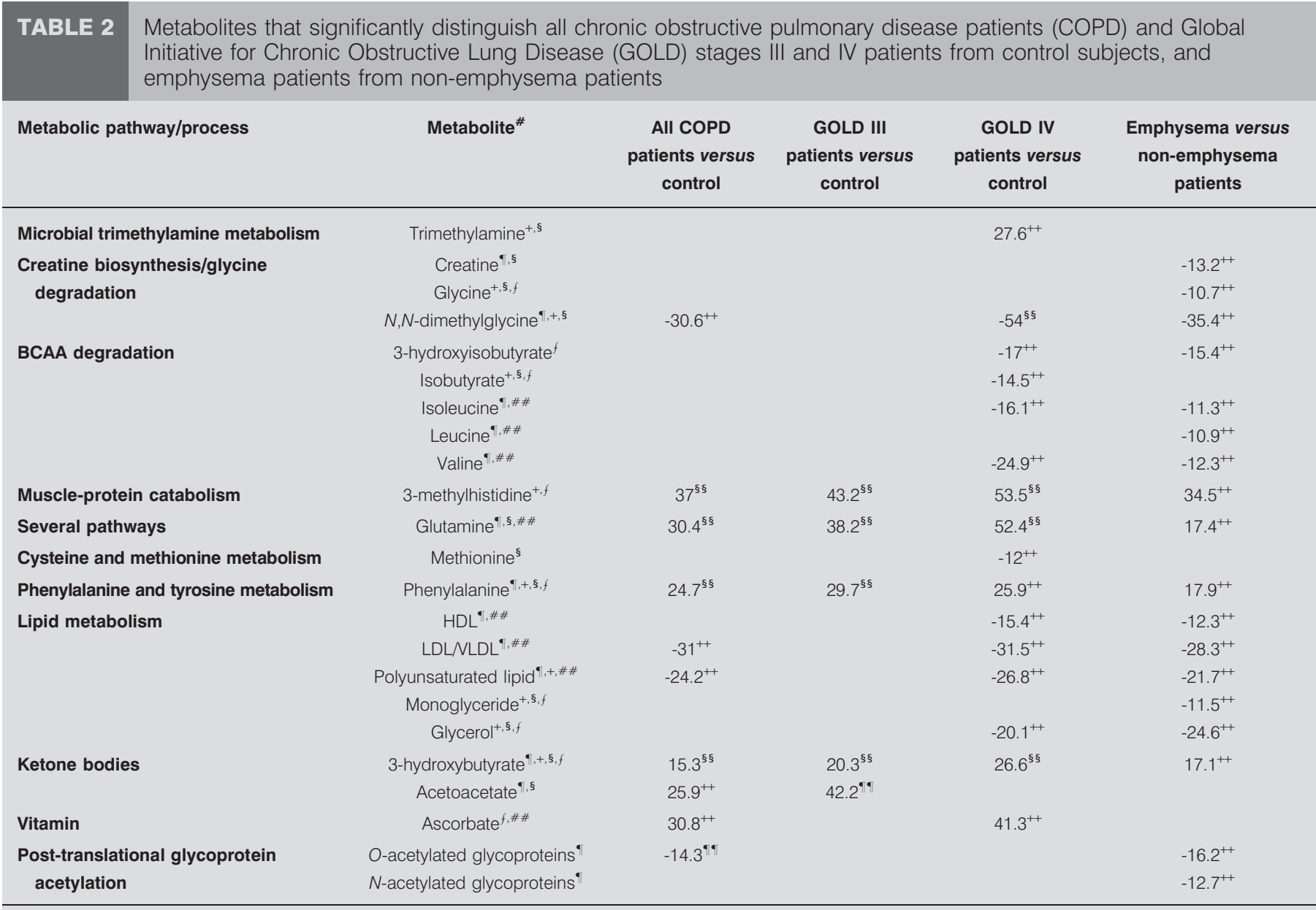

Data presented as \% positive or negative mean fold change (MFC). BCAA: branched-chain amino acid; HDL: high-density lipoproteins; LDL: low-density lipoproteins; VLDL: very low-density lipoproteins. ${ }^{\#}$ : unassigned metabolites also discriminated between each of the patient groups, the largest effect being an MFC of $106 \%$ (more information is included in the online supplementary material). ${ }^{\bullet}$ : assigned from the literature of TUKIAINEN and co-workers [10, 11]. ${ }^{+}$: assigned from GlaxoSmithKline database of standards (unpublished data). ${ }^{\varsigma}$ : assigned using Chenomx software. ${ }^{f}$ : assigned from the Human Metabolome Database. ${ }^{\# \#}$ : assigned from two-dimensional nuclear magnetic resonance correlation spectroscopy, total correlation spectroscopy, heteronuclear single-quantum correlation, and heteronuclear multiple-bond correlation on selected GOLD stage IV and control subjects' samples. The t-test raw p-value thresholds corresponding to $\alpha 0.05,0.1$ and 0.15 for each group were: GOLD III male and female $p<0.0013, p<0.0048$ and $p=0.0075$, respectively; GOLD IV male and female $p<0.0031, p<0.011$ and $p=0.0335$, respectively; all COPD patients (i.e. GOLD II, III, and IV male and female) $p<0.0013, p<0.0151$ and $p=0.0266$, respectively; combined GOLD III and IV male and female versus control $p<0.0027, p<0.0110$ and $p=0.0250 .{ }^{~}$ : false discovery rate (FDR) $\alpha<0.15 ;{ }^{++}$: FDR $\alpha<0.10 ;{ }^{\S \S}$ : FDR $\alpha<0.05$.

remained "unseen" by the model. To add confidence that the results would be repeatable the PLS-DA models were tested with the remaining independent "unseen" data. Using the joint training model of GOLD stages III and IV versus control $(\mathrm{n}=90)$, $82 \%$ of new independent test samples $(n=71)$ were classified correctly into either COPD patient or control subjects (fig. 2).

\section{Relationship between serum metabolites and emphysema}

Emphysema was assessed by a CT scan and described as the percentage of lung pixels with low attenuation (LAA) values $<-950 \mathrm{HU}$ (see online supplementary material for methodological detail). The relationship of NMR data to emphysema (defined by LAA) was calculated using two different approaches. The first used LAA as a category to split the data into two groups defining the presence (LAA $>20 \%$ ) or absence (LAA $0-10 \%$ ) of emphysema, which were then analysed by
PLS-DA and t-tests. The second used LAA as a response variable that measured the extent of emphysema correlated with NMR data using PLS and Pearson's correlation.

Significant PLS-DA models and t-tests showed that BCAAs, aspartate, asparagine, glycine, creatine, $N, N$-dimethylglycine, glycerol, lipids (VLDL, LDL and HDL), $\mathrm{O}$ - and $\mathrm{N}$-acetylglycoproteins, and 3-hydroxyisobutyrate were significantly decreased in emphysema patients, and that 3-methylhistidine, phenylalanine, glutamine and 3-hydroxybutyrate were found to have increased (table 2 and fig. 3). No significant models were obtained when control subjects were excluded, possibly due to the reduced power $(n=121$ versus $n=254$ with controls, respectively).

Emphysema severity was defined in PLS models by increasing glutamine, betaine and 3-hydroxybutyrate, and decreasing 

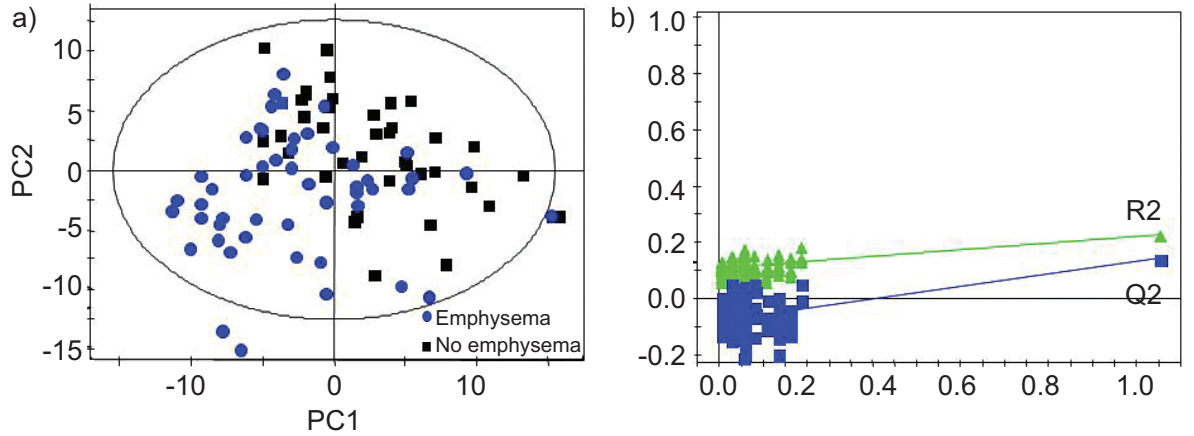

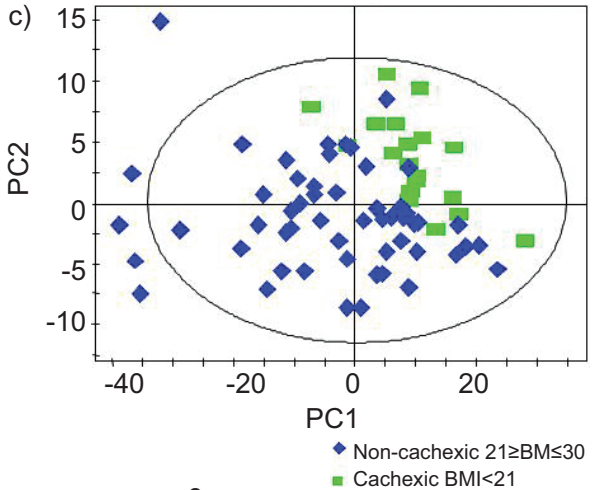

-Control
OGOLD IV

d)

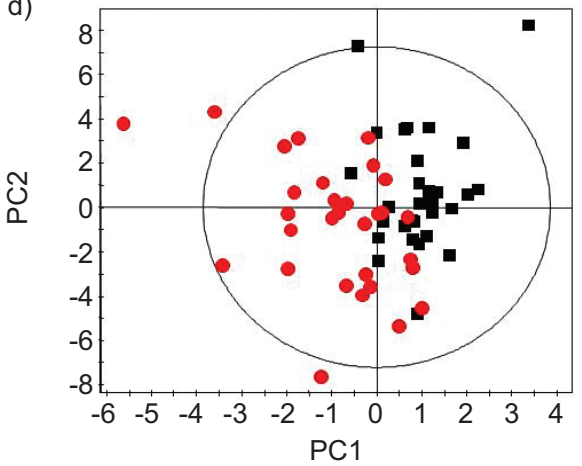

e)

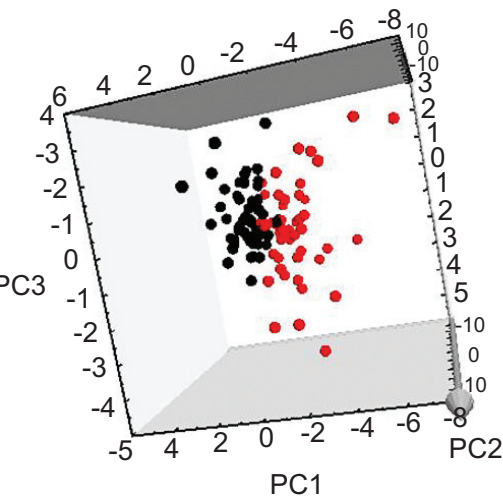

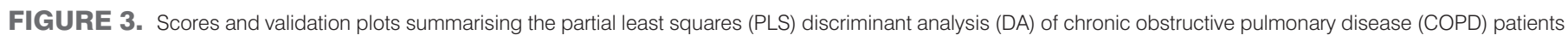

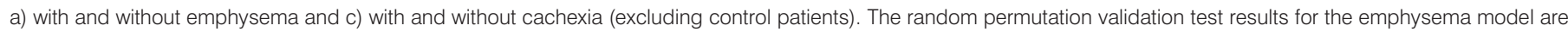

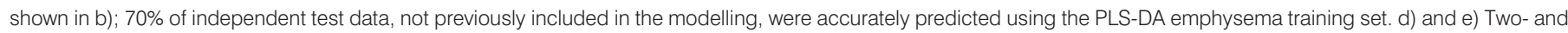

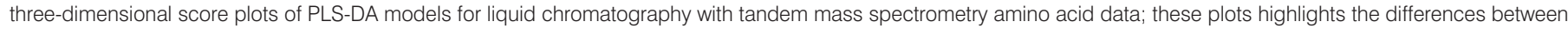
smoker control and GOLD stage IV; R2 $=45 \%, Q 2=26 \%$. All data were normalised according to serum creatinine levels.

glycerol and O-acetylglycoproteins. Significant Pearson's correlation coefficients with emphysema included 3-methylhistidine, glutamine, 3-hydroxybutyrate, $\alpha$-ketoadipate and scyllo-inositol (positive correlation) and BCAAs, alanine, glycine, guanidinoacetate, dimethylglycine, histidine, lipids, glucose, glycerol, 3-hydroxyisobutyrate, isobutyrate, and $\mathrm{O}$ - and $\mathrm{N}$-acetylated glycoproteins (negative; tables 3 and S3).

\section{Relationship between serum metabolites and systemic inflammation}

NMR data showed significant Pearson correlation coefficients with plasma C-reactive protein (CRP) and fibrinogen in patient and control groups. Fibrinogen levels were positively correlated with creatinine and log-transformed CRP levels with dimethylamine, 3-methylhistidine, glycine, creatinine, acetate, 3-aminoisobutyrate, 3-hydroxybutyrate and cis-aconitate in the patient group but not in the control group (table 3). PLS multivariate modelling highlighted that phenylalanine, histidine, alanine, $\mathrm{N}$-acetylglycoproteins, glutamine, aspartate, 3-hydroxybutyrate, creatine, glycerol, citrate and valine predicted levels of CRP and fibrinogen, and hence are most closely associated with increased systemic inflammation.

Both plasma CRP and fibrinogen were significantly altered between control and the different GOLD stages (ANOVA p-values were $2.55 \times 10^{-5}$ and $4.5 \times 10^{-5}$ for log CRP and fibrinogen, respectively). Post hoc t-test analysis of the individual group comparisons showed that CRP was significantly different between former smoker controls and GOLD stages II ( $p=$ $0.0014)$, III $(p=0.0008)$ and IV $(p=0.0005)$. Fibrinogen levels were significantly different between control and GOLD stages III $(p=0003)$ and IV $(p=5.71 E-05)$, and also between GOLD stages II and III $(p=0.0127)$ and IV $(p=0.0021)$, but not between control and GOLD stage II $(\mathrm{p}=0.0637)$.

\section{Relationship between serum metabolites and body mass index}

PCA of the full dataset showed a body mass index (BMI)related pattern in the score plot of $\mathrm{PC} 1$ and $\mathrm{PC} 2$, with cachexic subjects (BMI 15-21 $\mathrm{kg} \cdot \mathrm{m}^{-2}$ ) clustering separately from extremely obese subjects $\left(\mathrm{BMI}>40 \mathrm{~kg} \cdot \mathrm{m}^{-2}\right.$ ) due to reduced serum levels of acetylated glycoproteins and BCAAs.

Significant Pearson correlation coefficients of NMR data with BMI and fat-free mass (FFM) were indicative of metabolites that depicted cachexia and body habitus. COPD-specific effects of cachexia, an extreme form of habitus, were determined by comparing correlation coefficients in patients with controls (table 3). Metabolites correlated with FFM for COPD patients but not controls were glycine, proline, glycerol, and creatine (negative), and methylamine, creatinine, $N, N$-dimethylglycine and polyunsaturated lipid (positive). Metabolites that were correlated to BMI in patients but not controls were glutamine, HDL, N-methylnicotinate and ascorbate (table 3). 


\begin{tabular}{|c|c|c|c|c|c|c|c|}
\hline \multirow[t]{2}{*}{ Biochemical pathway/process } & \multicolumn{2}{|l|}{ BMI } & \multicolumn{2}{|c|}{ FFM } & \multirow[t]{2}{*}{ CRP Pos } & \multirow[t]{2}{*}{ Fibrinogen Pos } & \multirow[t]{2}{*}{ FV950 $^{\circ}$ Pos $^{+}$} \\
\hline & Pos & Neg & Pos & Neg & & & \\
\hline $\begin{array}{l}\text { His metabolism, muscle protein } \\
\text { catabolism }^{\mathbf{s}}\end{array}$ & & & & & $3 \mathrm{MH}$, His & & $3 \mathrm{MH}$ \\
\hline Cr biosynthesis/Gly degradation ${ }^{5}$ & Arg, Guan & & Cn, DMG & Gly, Cr & $\begin{array}{l}\text { Cr, Cn, } \\
\text { Gly, Guan, } \\
\text { Bet, Ch }\end{array}$ & $\mathrm{Cn}$ & \\
\hline Arg and Pro metabolism ${ }^{5}$ & $\operatorname{Arg}$ & & & Pro & & & \\
\hline Lys degradation ${ }^{5}$ & & & & & Lys, AKA & & AKA \\
\hline $\begin{array}{l}\text { Energy metabolism including } \\
\text { glycolysis, gluconeogenesis }\end{array}$ & $\begin{array}{l}\text { Lac, Pyr, } \\
\text { Glc }\end{array}$ & & & & Glc, Cac, & & \\
\hline and TCA cycle & & & & & Cit, Ac & & \\
\hline Ketone bodies & $3 \mathrm{HB}$ & & & & $\begin{array}{l}\text { 3HIB, AcAc, } \\
\quad \text { Aco }\end{array}$ & & $3 \mathrm{HIB}$ \\
\hline Nicotinate/nicotinamide metabolism & & NMA & & & & & \\
\hline Acetylated glycoproteins & OAc, NAc & & OAc, NAc & & OAc, NAc & NAc & \\
\hline Vitamins & Asc & & & & Myo & Asc & Scyllo \\
\hline
\end{tabular}

No negative correlations were observed with CRP or fibrinogen. BMI: body mass index; FFM: fat-free mass; Pos: positive; Neg: negative; BCAA: branched-chain amino acids; Cr: creatine: Gly: glycine; Phe: phenylalanine; Tyr: tyrosine; Ala: alanine; Asp: aspartate; Glu: glutamate; Gln: glutamine; Arg: arginine; Pro: proline; Lys: lysine; TCA: tricarboxylic acid; TMA: trimethylamine; Val: valine; Leu: leucine; lle: isoleucine; 3HIB: 3-hydroxyisobutyrate; ISB: isobutyrate; Guan: guanidinoacetate; Lac: lactate; Pyr: pyruvate; Glc: glucose; 3HB: 3-hydroxybutyrate; OAc: O-acetylglycoprotein; NAc: N-acetylglycoprotein; Asc: ascorbate; HDL: high-density lipoprotein; LDL: lowdensity lipoprotein; VLDL: very low-density lipoprotein; TG: triglyceride; MG: monoglycerides; Poly: polyunsaturated lipid; Glycl: glycerol; NMA: N-methylnicotinic acid; Cn: creatinine; DMG: N,N-dimethylglycine; 3MH: 3-methylhistidine; His: histidine; 3AIB: 3-aminoisobutyrate; Bet: betaine; Ch: choline; PAG: phenylacetylglycine; AKA: $\alpha$-ketoadipate; Cac: cis-aconitate; Cit: citrate; Ac: acetate; AcAc: acetoacetate; Aco: aconitate; Myo: myo-inositol; PC: phosphorylcholine; Form: formate; Scyllo: scylloinositol; form: formate. ${ }^{\#}$ : full data including correlation coefficient values are shown in table S5; ": percentage of lung pixels with low attenuation on the whole lung scans with attenuation values of $<-950 \mathrm{HU} ;{ }^{+}$: higher FV950 values indicated greater emphysema; ${ }^{\text {s: }}$ amino acid metabolites generated and utilised during protein synthesis and degradation.

PLS modelling showed that increased VLDL, unsaturated lipid, glucose, valine, lactate, alanine, pyruvate, guanidinoacetate, glutamate and $\mathrm{O}$-acetylated glycoproteins, and reduced glycine, glutamine, 3-hydroxybutyrate, phenylalanine, methionine, acetone, acetate, betaine, lysine, proline, aspartate and alanine (table 3) predicted BMI. In addition, increased BCAAs, creatinine, alanine, glutamate, betaine, acetone and $\mathrm{O}$-acetylated glycoproteins, and decreased glycine, creatine, HDL, VLDL, glycerol, monoglycerides and acetate predicted FFM (tables 4, S4 and S5).

PLS-DA modelling of cachexic (BMI $<21 \mathrm{~kg} \cdot \mathrm{m}^{-2}$ ) versus noncachexic patients (BMI $21-30 \mathrm{~kg} \cdot \mathrm{m}^{-2}$ ) showed that in cachexic patients, glutamine, glycine, 3-hydroxybutyrate, acetate, methionine and 3-aminoisobutyrate were increased, and valine, isoleucine, ascorbate, pyruvate and glucose were decreased.

\section{Preliminary validation of amino acid changes using LC-MS/MS}

Open-profiling metabolomics showed that several metabolites differed between control and COPD patient subgroups. In order to validate the amino acid changes analytically using a more sensitive and quantitative approach, targeted metabolomic experiments using LC-MS/MS were developed. The LC-MS/ MS amino acid assay was then applied to a subset of the original samples of the most severely affected male patients (GOLD 


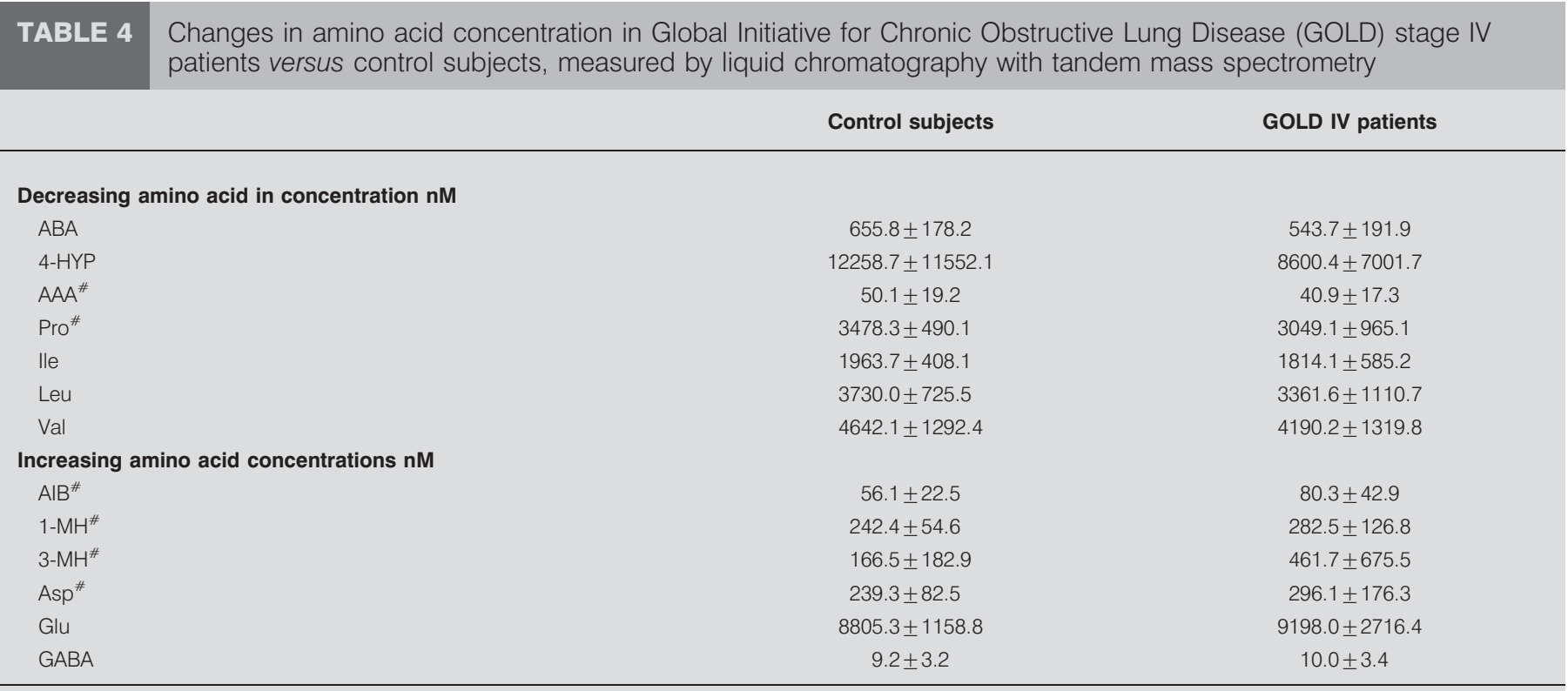

Data are presented as mean \pm SD. ABA: $\alpha$-aminobutyrate; 4-HYP: 4-hydroxyproline; AAA: aminoadipate; Pro: proline; lle: isoleucine, Leu: leucine; Val: valine, AIB: aminoisobutyrate; 1-MH: 1-methyhistidine; 3-MH: 3-methylhistidine; Asp: aspartate; Glu: glutamine; GABA: $\gamma$-aminobutyrate. Principal component (PC)1 and PC2 $R^{2}=37.5 \%$ and $Q^{2}=26.2 \%$; PC3-PC5 $R^{2}=63.9 \%, Q^{2}=36.3$. ${ }^{*}$ : amino acids with $p$-values $<0.1$ (adjusted for using Benjamini-Hochberg test, false discovery rate $\alpha=0.1$ ).

stage IV; $\mathrm{n}=30$ ) compared to controls (ex-smoker; $\mathrm{n}=30$ ). A significant PLS-DA model (fig. 3; PC1 $\mathrm{R}^{2}=37.5 \%$ and $\mathrm{Q}^{2}=$ $26.2 \%$ ) highlighted aminoisobutyrate as most predictive of GOLD stage IV versus control (table 4). BCAA coefficients were also high for the model, consistent with NMR findings, although absolute values did not show significant separation using t-tests.

\section{DISCUSSION}

Non-targeted metabolomics is well suited for biomarker discovery, allowing investigation of many molecules, with diverse chemical structures, simultaneously. The investigation of multiple markers facilitates the potential discovery of biomarker panels, which are more likely to be necessary than single entities to describe systemic effects of COPD. We have applied metabolomics to ECLIPSE serum samples to assist in the drive to discover biomarkers that may reflect patient outcome more predictively than FEV1, which is currently used. Because ECLIPSE is the largest study currently attempting to better describe the subtypes of COPD [4], we have had unprecedented access to substantial amounts of patient information related to the characterisation of disease status, body composition, serum biochemistry, medication use and food intake. This has enabled us to put metabolomic data in context with the patient and look for associations that have not been possible previously ( $n=244$, total number of serum samples in the dataset). Our results have confirmed that serum metabolomic profiles of COPD patients correlate with different characteristics of COPD severity assessed by spirometry, body composition, emphysema and systemic inflammation.

The use of independent test sets for PLS-DA models (i.e. data not used to generate the model), together with the observation of patient groupings in unsupervised PCA analysis and the application of FDR to t-test results reduce the chance of making a false-positive discovery (i.e. type one statistical error), giving extra confidence in the statistical results. Without these tests the nature of all statistical tests indicates that at the 5-15\% significance level, used in these studies, $15 \%$ of all the variables tested (i.e. $\geqslant 90$ ) would be assigned as significant, even in a random dataset with no real significant effects.

\section{Serum metabolite differences between COPD patients and control subjects}

Because COPD-related effects were greater than variation due to sex, age, geographical location or any other potentially confounding factors, joint male and female models were used in analysis. Metabolomic profiles for all COPD patients depicted reduced food intake (decreased VLDL, LDL and chylomicrons), increased utilisation of ketone bodies as an alternative energy source (3-hydroxybutyrate) and decreased post-translational protein modification/protein synthesis (acetylated glycoproteins). All COPD patients also showed increased concentrations of serum methylhistidine, an amino acid formed during crosslinking of muscle protein synthesis, suggesting increased muscle actin and myosin protein degradation [12], even in patients that were not cachexic.

\section{Relationship between serum metabolites and disease severity}

As with many inflammatory markers, particularly markers of systemic inflammation, significant changes did not appear until more severe disease stages (GOLD III and IV) with no correlation or significant models observed for the GOLD stage II group. In severely affected patients (GOLD stage IV), increased degradation of muscle protein (3-methylhistidine) and utilisation of BCAAs as an alternative source of energy for muscle and adipose tissue were indicated.

Several findings are reflective of altered protein metabolism (methylhistidine, BCAAs and their degradation product 
3-hydroxyisobutyrate) associated with disease as well as the extent of disease. As well as being correlated with BMI, COPD patients, independent of cachexia, have increased levels of glutamine and ketone bodies, and decreased VLDL/LDL and O-acetylglycoproteins.

Relationship between serum metabolites and emphysema In individuals with emphysema, increased degradation of muscle protein (3-methylhistidine) and utilisation of BCAAs were indicated. Increased serum methylhistidine in patients with emphysema was not correlated to either BMI or FFM, suggesting that increased muscle protein turnover is a feature of COPD related to emphysema that precedes or is unrelated to the development of cachexia.

Disrupted creatine synthesis and glycine degradation occurred in this group, with lower serum levels of many intermediates (table 2 and 3 ).

\section{Relationship between serum metabolites and systemic inflammation}

For individuals experiencing high systemic inflammation (higher CRP levels) increased degradation of muscle protein (3-methylhistidine) and utilisation of BCAAs were indicated.

\section{Relationship between serum metabolites and BMI}

Cachexia, defined as low BMI and FFM, was characterised more extensively by protein muscle breakdown and decreased BCAAs and their degradation products. The metabolomic results can be subdivided into those that reflect general changes in body habitus and those that reflect distinct differences in COPD patients. Correlations between NMR data and BMI and FFM in controls indicate that many cachexia-related changes are also associated with altered general habitus (i.e. BMI changes; table S4). These include positive correlation with BCAAs, alanine, creatinine, choline, betaine, VLDL/LDL, ketone bodies, acetate, 3-hydroxyisobutyrate, citrate, lactate, pyruvate, glycerol and acetylated glycoproteins. The PLS-DA model of cachexia versus non-cachexia and Pearson correlations in the patient group show that some metabolites are only altered in COPD patients and, therefore, are not reflective of general habitus (HDL, ascorbate, $\mathrm{N}$-methylnicotinate, glutamine (negative correlation)).

\section{Preliminary validation of amino acid changes using LC-MS/MS}

The LC-MS/MS targeted amino-acid profiling served as a preliminary analytical validation study of the NMR results. The main role of this study was to explore whether there was consistency between both analytical platforms prior to extension of the targeted amino acid study to the full sample set. This was successful using LC-MS/MS of male GOLD stage IV patients versus control subjects, where MS results were in agreement with NMR results within the different limitations and detection limits for each platform (tables 2 and S5). The reduced power of the small study meant that whilst trends were observed in the amino acids detected by NMR only one of these was consistent after accounting for repeat measures. A batch and run order effect was also observed in the full sample set; methods of elimination of run order and batch effects for MS data are currently being explored and the analysis of this dataset will be reported separately.

\section{Biochemical role of metabolite changes and possible implication for ECLIPSE patients}

Decreased BCAAs have been found in previous studies of COPD patients [13-15]. BCAAs regulate protein turnover and glucose homeostasis [16] with a continuous flux of BCAA to skeletal muscle. Skeletal muscle produces $90 \%$ of total glutamine [16], which in turn is a substrate for proliferative cells including immune cells [17]. Muscle proteolysis and transamination of BCAAs via branched-chain aminotransferase is part of an essential physiological function to provide carbon for gluconeogenesis during fasting. Cachexic patients who are losing weight demonstrate increased rates of gluconeogenesis not suppressed by glucose [18]; BCAAs may represent a physiological response to weight loss, consistent with the strong correlation of BCAA levels with BMI in data from the ECLIPSE cohort.

Approximately 25\% of COPD patients develop cachexia, which is associated with a poor prognosis [19]. Cachexia is characterised by altered BCAA availability, due partly to anorexic effects of COPD, i.e. low food intake accompanied by elevated oxidation of BCAAs and gluconeogenesis. Protein degradation to increase BCAAs supply and glucogenic amino acids occur at the same time as suppression of protein synthesis. Changes observed in the NMR data, i.e. reduced BCAAs and increased glutamine and alanine are consistent with cachexia/increased protein degradation within this patient group. Increases in other essential amino acids including phenylalanine were consistent with increased protein degradation. Consistently altered 3-methylhistidine suggests increased muscle protein turnover precedes or is independent of the onset of cachexia.

In this study, metabolites that correlated strongly with cachexia markers also correlated strongly with systemic inflammation. In fasting and starving states [20], short-term levels of BCAAs rise in parallel with increased protein degradation. After several weeks, the rate of muscle protein degradation decreases and, therefore, blood concentration of BCAAs decreases as ketone-body production increases. Metabolomic data for COPD patients showed increased ketone bodies and decreased BCAAs, and in emphysema patients there was also a decrease in serum creatine and its precursors, glycine, guanidinoacetate and dimethylglycine (fig. 4). This suggests a reduced mitochondrial function, consistent with recent reports in the early stages of COPD where skeletal muscle mitochondria show an electron transport chain blockade and excessive production of reactive oxygen species [21].

\section{Further work}

Serum and urine are available from each time-point in the ECLIPSE study. Expansion of the current work will include investigation of serum samples longitudinally to determine which markers track with disease progression and are most successful at prediction of patient outcome. These studies will include LC-MS/MS amino acid targeted analysis to obtain quantitative values for biomarkers of interest and exploration of any sex bias of significant effects. Urine data have already been acquired for the baseline time-point; open-profiling NMR and LC-MS results will be reported elsewhere. The combination of metabolomics data with other types of gene transcript, genetic and protein data at both the numerical and pathway level is in progress to investigate significant pathways involved with COPD. 

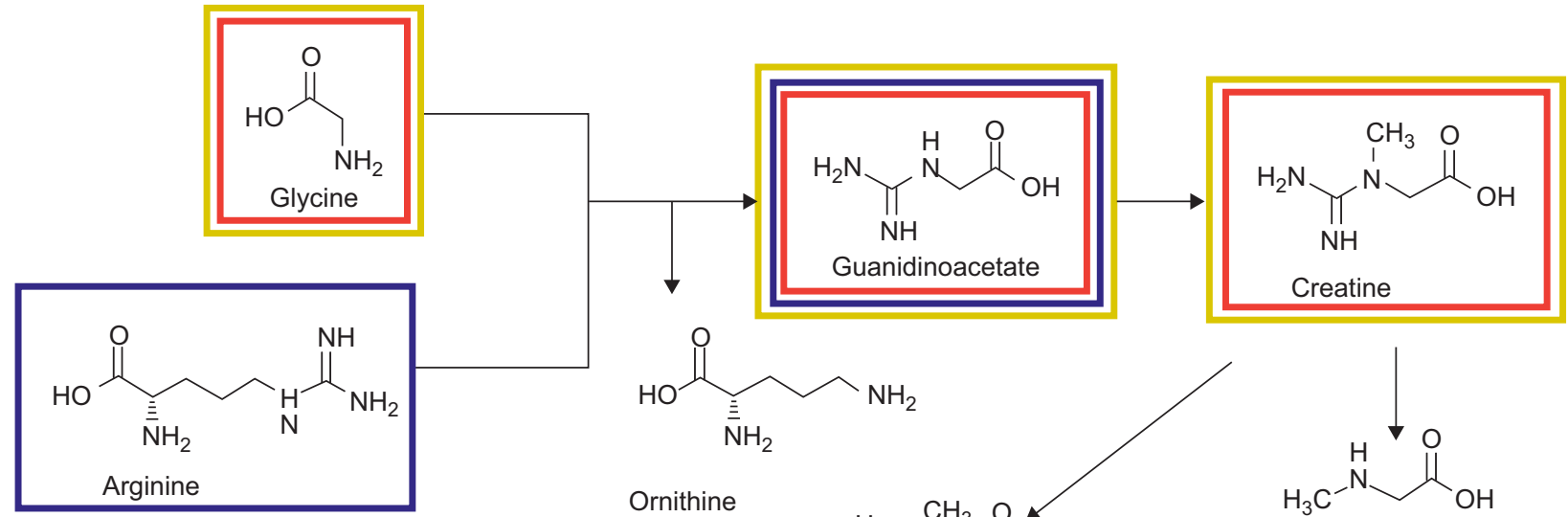<smiles>NCCC[C@H](N)C(=O)O</smiles>
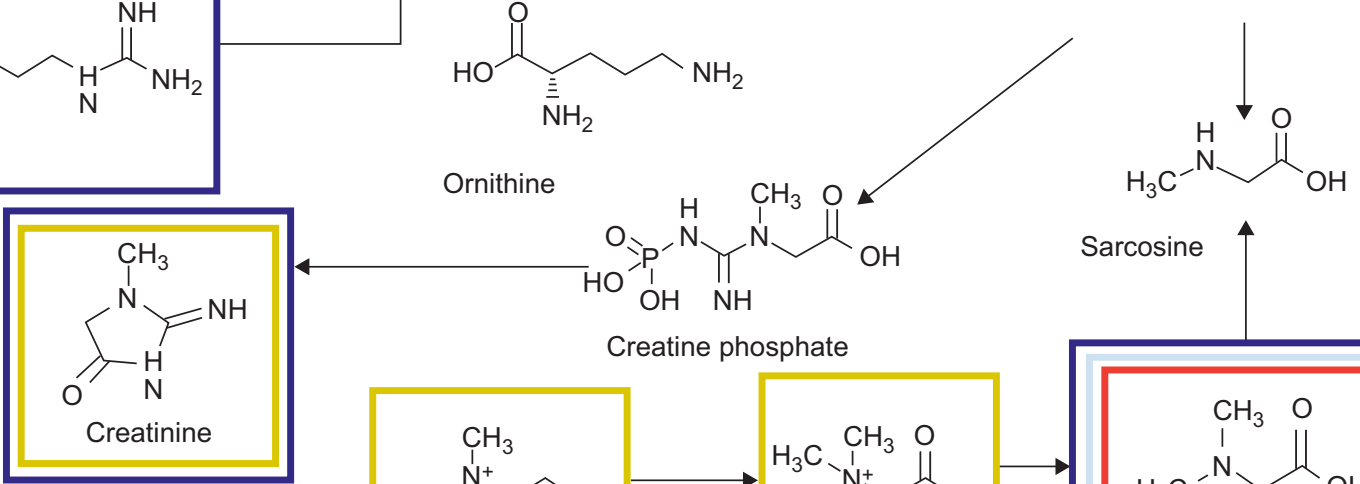

Ornithine

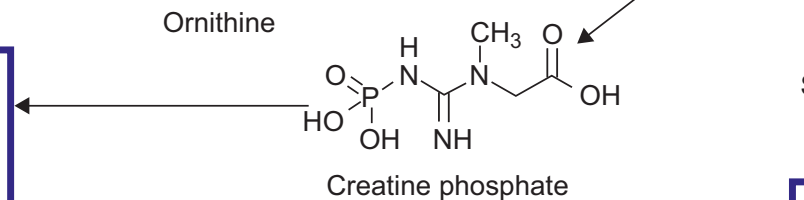

Sarcosine

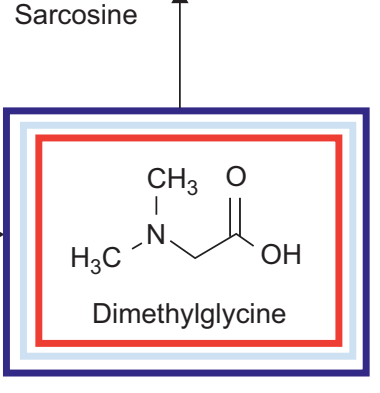

Reduced in emphysema patients

Reduced in patient versus control

Positive correlation with BMI/FFM

Positive correlation with CRP/fibrinogen

FIGURE 4. Creatine biosynthesis and glycine degradation pathway showing metabolites that were altered in chronic obstructive pulmonary disease patients. Each arrow represents one step in the pathway. BMI: body mass index; FFM: fat-free mass; CRP: C-reactive protein.

Ultimately, biomarker validation studies in other patient cohorts and investigation of normal variation of candidate biomarkers in human epidemiological studies are required. It is also planned to explore the different metabolomics profiles and proposed biomarker panels in relation to different drug treatments.

\section{Conclusions}

We have applied metabolomics to the analysis of serum samples from ECLIPSE patients. ECLIPSE is the largest COPD study of its type and, as such, we have had access to patient characterisation on a much larger scale than previous metabolomics studies of COPD. Due to the increased power of the experiments, we have been able to characterise metabolomics profiles characteristic of cachexia, inflammation, emphysema and disease severity, as well as distinguishing spectra from patients and controls. Thus, we have demonstrated that NMR-based metabolomics provides additional phenotypic information in COPD patients based on a combination of their systemic effects and lung-function parameters. The main effects suggest increased protein turnover in all COPD patients together with increased protein degradation in emphysema and cachexic patients. Metabolic evidence of reduced food intake was observed in COPD patients, which correlated with reduced BMI. Evidence of reduced mitochondrial oxidation efficiency was also indicated by the correlation of ketone bodies with clinical parameters. A subset of these markers (e.g. BCAAs, methylhistidine, phenylalanine, acetylated glycoproteins, 3-hydroxypyruvate, serum lipid and ascorbate) could provide a useful biomarker panel by which to assess and stratify COPD patients according to their protein turnover, mitochondrial function and nutritional status.

\section{SUPPORT STATEMENT}

This study was funded by GlaxoSmithKline.

\section{STATEMENT OF INTEREST}

Statements of interests for B.K. Ubhi, J.H. Riley, P.A. Shaw, D.A. Lomas, R. Tal-Singer, W. MacNee, S.C. Connor and the study itself can be found at www.erj.ersjournals.com/site/misc/statements.xhtml

\section{ACKNOWLEDGEMENTS}

The authors would like to thank the Depts of Sample Management, Statistical Sciences, and Investigative Preclinical Toxicology at GlaxoSmithKline (Stevenage and Ware, respectively, UK) for technical assistance and scientific input. We would also like to thank B.C. Sweatman (investigative Preclinical Toxicology Dept, GlaxoSmithKline, Ware, UK) for NMR analysis and S.M. Fox (Biostatistics, Stevenage Medicines Development, GlaxoSmithKline, Stevenage, UK) for statistical advice.

\section{REFERENCES}

1 Buist AS, McBurnie MA, Vollmer WM, et al. International variation in the prevalence of COPD (the BOLD study): a population-based prevalence study. Lancet 2007; 370: 741-750.

2 Lopez AD, Shibuya K, Rao C, et al. Chronic obstructive pulmonary disease: current burden and future projections. Eur Respir J 2006; 27: $397-412$ 
3 Barton RH. A decade of advances in metabonomics. Expert Opin Drug Metab Toxicol 2011; 7: 129-136.

4 Vestbo J, Anderson W, Coxson HO, et al. Evaluation of COPD Longitudinally to Identify Predictive Surrogate End-points (ECLIPSE). Eur Respir J 2008; 31: 869-873.

5 Agusti A, Calverley PMA, Celli B, et al. Characterisation of COPD heterogeneity in the ECLIPSE cohort. Respir Res 2010; 11: 122-136.

6 Pauwels RA, Buist AS, Calverley PMA, et al. Global strategy for the diagnosis, management, and prevention of chronic obstructive pulmonary disease. NHLBI/WHO Global Initiative for Chronic Obstructive Lung Disease (GOLD) Workshop Summary. Am J Respir Crit Care Med 2001; 163: 1256-1276.

7 Alum MF, Shaw A, Sweatman BC, et al. 4,4-Dimethyl-4silapentane-1-ammonium trifluoroacetate (DSA), a promising universal internal standard for NMR-based metabolic profiling studies of biofluids, including blood plasma and serum. Metabolomics 2008; 4: 2 122-127.

8 Simpson JH. Organic Structure Determination using 2-D NMR Spectroscopy: A Problem-Based Approach (Advanced Organic Chemistry). Burlington, Academic Press, 2008.

9 Benjamini Y, Hochberg Y. Controlling the false discovery rate: a practical and powerful approach to multiple testing. J R Statist Soc Series B 1995; 57: 289-300.

10 Tukiainen T, Tynkkynen T, Mäkinen VP, et al. A multi-metabolite analysis of serum by $1 \mathrm{H}$ NMR spectroscopy: early systemic signs of Alzheimer's disease. Biochem Biophys Res Commun 2008; 375: 356-361.

11 Nicholson JK, Foxall PJD, Spraul M, et al. $750 \mathrm{MHz} 1 \mathrm{H}$ and $1 \mathrm{H}-13 \mathrm{C}$ NMR spectroscopy of human blood plasma. Anal Chem 1995; 67: 793-811.
12 Young VR, Munro HN. N $\tau$-methylhistidine (3-methylhistidine) and muscle protein turnover: an overview. Fed Proc 1978; 37: 2291-2300.

13 Hofford JM, Milakofsky L, Vogel WH, et al. The nutritional status in advanced emphysema associated with chronic bronchitis. A study of amino acid and catecholamine levels. Am Rev Respir Dis 1990; 141: 902-908

14 Schols AMWJ, Duetz NEP, Mostert R, et al. Plasma amino acid levels in patients with chronic obstructive pulmonary disease. Monaldi Arch Chest Dis 1993; 48: 546-548.

15 Engelen MPKJ, Wouters EFM, Duetz NEP, et al. Factors contributing to alterations in skeletal muscle and plasma amino acid profiles in patients with chronic obstructive pulmonary disease. Am J Clin Nutr 2000; 72: 1480-1487.

16 Layman DL, Walker DA. Branched chain amino acids: metabolism, physiological function and application. J Nutrition 2006; 136: $319 \mathrm{~S}$.

17 Thompson D. Cancer: genes, cachexia and death. In: Newsholme E, Leech T, eds. Functional Biochemistry in Health and Disease. Chichester, John Wiley \& Sons Ltd, 2010; pp. 485-507.

18 Baracos VE, Mackenzie ML. Investigations of branched-chain amino acids and their metabolites in animal models of cancer. J Nutri 2006; 136: 237S-242S.

19 Wagner PD. Possible mechanisms underlying the development of cachexia in COPD. Eur Respir J 2008; 31: 492-501.

20 Tom A, Nair S. Assessment of branched-chain amino acids and potential for biomarkers. J Nutri 2006; 136: 324S-330S.

21 Puente-Maestu L, Pérez-Parra J, Godoy R, et al. Abnormal mitochondrial function in locomotor and respiratory muscles of COPD patients. Eur Respir J 2009; 33: 1045-1052. 